\title{
Principal models on a solvable group with nonconstant metric
}

\author{
LADislav HLAVATÝ * \\ Department of Physics, \\ Faculty of Nuclear Sciences and Physical Engineering
}

November 13, 2018

\begin{abstract}
Field equations for generalized principle models with nonconstant metric are derived and ansatz for their Lax pairs is given. Equations that define the Lax pairs are solved for the simplest solvable group. The solution is dependent on one free variable that can serve as the spectral parameter. Painlevé analysis of the resulting model is performed and its particular solutions are found
\end{abstract}

1991 MSC numbers: 35L10, 35L15, 34A55

Keywords: principal models, chiral models, sigma models, Lax pair, integrable models, solvable group.

\section{Introduction}

The Lax pair formulation for principal models with the trivial $\left(\delta_{i j}\right)$ metric was published in [1] and Lax formulation of related $O(N)$-sigma

\footnotetext{
*Postal address: Břehová 7, 11519 Prague 1, Czech Republic. E-mail: hlavaty@br.fjfi.cvut.cz
} 
models in [2], 3]. The models with the nontrivial but constant metric on the group manifold were investigated e.g. in [ [- $-[5]$. The goal of this paper is to present an example of the principal model with a nonconstant metric on the group manifold having the Lax pair. The models with the nonconstant metrics we are aware of (e.g. [6], [7]) are different from those investigated in this paper.

In the previous work 8] we have given several conditions on the metric for $S U(2)$ models but we were not succesful in finding an example of the metric that is not constant on the group manifold and admits the Lax formulation of field equations. Trying to find such an example, we have chosen much simpler group, namely the group of afine transformations of the real line - denoted here $A f(1)$. It is a nonabelian solvable two-dimensional Lie group that can be realized as the group of matrices

$$
g=\left(\begin{array}{ll}
a & b \\
0 & 1
\end{array}\right), a>0, b \in \mathbf{R} .
$$

It is diffeomorphic to $\mathbf{R}^{2}$ and one can choose the global coordinates $\theta_{1}, \theta_{2}$ on $A f(1)$ setting e.g. $a=\exp \left(\theta_{1}\right), b=\theta_{2}$. The basis in the corresponding two-dimensional solvable Lie algebra can be chosen that the only nonvanishing structure coefficients are

$$
c_{12}^{2}=-c_{21}^{2}=1
$$

The coordinates of the left-invariant fields $J_{\mu}:=g^{-1} \partial_{\mu} g$ in this basis are $\left(\partial_{\mu} \theta_{1}, e^{-\theta_{1}} \partial_{\mu} \theta_{2}\right)$.

\section{Formulation of generalized principal chiral models}

Principal chiral models are given by the action

$$
I[g]=\int d^{2} x \eta^{\mu \nu} L\left(J_{\mu}, J_{\nu}\right)
$$

where

$$
J_{\mu}:=\left(g^{-1} \partial_{\mu} g\right) \in \mathcal{L}(G),
$$

$g: \mathbf{R}^{2} \rightarrow G, \mu, \nu \in\{0,1\}, \eta:=\operatorname{diag}(1,-1)$ and $L$ is the Killing form on the corresponding Lie algebra $\mathcal{L}(G)$. 
An immediate generalization of the principal chiral models is obtained when one considers a general invertible bilinear form instead of the Killing [9],[5]. Next step of generalization (3) is introducing $G$-dependent symmetric bilinear forms. The generalized principal chiral models are then defined by the action

$$
I[g]=\int d^{2} x L_{a b}(g) \eta^{\mu \nu}\left(g^{-1} \partial_{\mu} g\right)^{a}\left(g^{-1} \partial_{\nu} g\right)^{b},
$$

where $L_{a b}(g)$ is invertible symmetric matrix $\operatorname{dim} G \times \operatorname{dim} G$ defined by the $G$ - dependent bilinear form $L(g)$ as

$$
L_{a b}(g):=L(g)\left(t_{a} \otimes t_{b}\right),
$$

and $t_{j}$ are elements of a basis in the Lie algebra of the left-invariant fields. It is useful to consider the bilinear form $L(g)$ as a metric on the group manifold. Lie products of elements of the basis define the structure coefficients

$$
\left[t_{a}, t_{b}\right]=c_{a b}{ }^{c} t_{c}
$$

and in the same basis we define the coordinates of the field $J_{\nu}$

$$
J_{\nu}=g^{-1} \partial_{\nu} g=J_{\nu}^{b} t_{b}
$$

that satisfy the Bianchi identities

$$
\partial_{\mu} J_{\nu}-\partial_{\nu} J_{\mu}+\left[J_{\mu}, J_{\nu}\right]=0
$$

Varying the action (5) w.r.t. $\eta:=g^{-1} \delta g$ we obtain equations of motion for the generalized principal chiral models

$$
\partial_{\mu} J^{\mu, a}+\Gamma_{b c}^{a} J_{\mu}^{b} J^{\mu, c}=0,
$$

where

$$
\Gamma_{b c}^{a}:=S_{b c}^{a}+\gamma_{b c}^{a},
$$

$S^{a}{ }_{b c}$ is the so called flat connection given by the structure coefficients

$$
S_{b c}^{a}:=\frac{1}{2}\left(C_{b c}^{a}+C_{c b}^{a}\right), C_{b c}^{a}:=\left(L^{-1}\right)^{a p} c_{p b}{ }^{q} L_{q c}
$$

and $\gamma_{b c}^{a}$ are the Christoffel symbols for the metric $L_{a b}(g)$ on the group manifold

$$
\gamma_{b c}^{a}:=\frac{1}{2}\left(L^{-1}\right)^{a d}\left(U_{b} L_{c d}+U_{c} L_{b d}-U_{d} L_{b c}\right) .
$$


The vector fields $U_{a}$ in (13) are defined in the local group coordinates $\theta_{i}$ as

$$
U_{a}:=U_{a}^{i}(\theta) \frac{\partial}{\partial \theta_{i}}
$$

where the matrix $U$ is inverse to the matrix $V$ of vielbein coordinates

$$
U_{a}^{i}(\theta):=\left(V^{-1}\right)_{a}^{i}(\theta), \quad V_{i}^{a}(\theta):=\left(g^{-1} \frac{\partial g}{\partial \theta_{i}}\right)^{a} .
$$

Note that the connection (11) is symmetric in the lower indices

$$
\Gamma_{b c}^{a}=\Gamma_{c b}^{a} .
$$

For the group $A f(1)$

$$
U_{a}=\left(\frac{\partial}{\partial \theta_{1}}, e^{\theta_{1}} \frac{\partial}{\partial \theta_{2}}\right)
$$

\section{The Lax pairs}

In the paper [5], the ansatz for the Lax formulation of the generalized chiral models was taken in the form

$$
\left[\partial_{0}+P_{a b} J_{0}^{b} t_{a}+Q_{a b} J_{1}^{b} t_{a}, \partial_{1}+P_{a b} J_{1}^{b} t_{a}+Q_{a b} J_{0}^{b} t_{a}\right]=0
$$

where $P, Q$ are two auxiliary $\operatorname{dim} G \times \operatorname{dim} G$ matrices. The ansatz (18) is a generalization of the Lax pair for Killing metric on the compact semisimple group $\mathrm{E}_{a b}=\operatorname{Tr}\left(t_{a} t_{b}\right)$ (in that case $Q$ and $P$ are multiples of the unit matrix).

Necessary conditions that the operators in (18) form the Lax pair for the equations of motion (10) are

$$
\begin{gathered}
\partial_{0} P=\partial_{1} Q, \quad \partial_{0} Q=\partial_{1} P \\
\left(P_{b p} P_{c q}-Q_{b p} Q_{c q}\right) c_{b c}{ }^{a}=P_{a b} c_{p q}{ }^{b} \\
\frac{1}{2} c_{c d}{ }^{a}\left(P_{c p} Q_{d q}+P_{c q} Q_{d p}\right)=Q_{a b} \Gamma_{p q}^{b} .
\end{gathered}
$$

If $Q$ is invertible, that we shall assume in the following, then these conditions are also sufficient. Note that the first two conditions are independent of the bilinear form $L$ so that one can start with solving the equation (20) and then look for the bilinear forms $L$ that admit 
solution of the equation (19,21). Moreover, as we have no a priori conditions for the first derivatives of the fields $g$ i.e. derivatives of $\theta_{1}, \theta_{2}$, we get from (19)

$$
\partial_{\theta_{j}} P=0, \partial_{\theta_{j}} Q=0
$$

\subsection{Solution of the equations (19-21) for $A f(1)$}

The equations (20 21) where $a=1$ become linear equations for elements $P_{1 b}$ and $Q_{1 b}$ due to the fact that $c_{p q}{ }^{1}=0$. It is rather easy to see that there is only trivial solution $Q_{11}=Q_{12}=0$ of the equations $Q_{1 b} \Gamma^{b}{ }_{p q}=0$ for constant nondegenerate metric $L$ (i.e. $\Gamma_{p q}^{b}=S^{b}{ }_{p q}$ ) so that there is no invertible matrix $Q$ that satisfy the equations (21) for $A f(1)$ and the constant nondegenerate metric $L$.

On the other hand, there are solutions of the equations (20-21) with $\operatorname{det} Q \neq 0$ for the nonconstant metric. In the following we shall present solutions for the diagonal metric

$$
L(g)=\left(\begin{array}{cc}
k_{1}\left(\theta_{1}, \theta_{2}\right) & 0 \\
0 & k_{2}\left(\theta_{1}, \theta_{2}\right)
\end{array}\right) .
$$

The equation (20) for $A f(1)$ imply that matrix $P$ is of the form

$$
P=\left(\begin{array}{cc}
p_{1} & 0 \\
p_{3} & p_{2}
\end{array}\right)
$$

where

$$
p_{2}\left(p_{1}+1\right)=\operatorname{det} Q
$$

The linear equations for the elements $Q_{1 b}$ given by (21) read

$$
\begin{gathered}
Q_{1 b} \gamma_{11}^{b}=0, \\
2 Q_{1 b} \gamma_{12}^{b}+Q_{12}=0, \\
k_{1} Q_{1 b} \gamma_{22}^{b}-k_{2} Q_{11}=0 .
\end{gathered}
$$

The condition $\operatorname{det} Q \neq 0$ requires that this system has a nontrivial solution. The nontrivial solvability of this system implies a system of quadratic equations for the Christoffel symbols that finally give a system of partial differential equations for the elements of the metric

$$
\left(k_{2}-k_{2,1}\right) k_{1,1}-e^{2 \theta_{1}} k_{1,2}^{2}=0
$$




$$
\begin{gathered}
\left(2 k_{2}-k_{2,1}\right) k_{1,2}+k_{1,1} k_{2,2}=0 \\
\left(k_{2}-k_{2,1}\right)\left(2 k_{2}-k_{2,1}\right)+e^{2 \theta_{1}} k_{1,2} k_{2,2}=0
\end{gathered}
$$

where $k_{i, j} \equiv \frac{\partial k_{i}}{\partial \theta_{j}}$. For every solution of this system we can get a solution of the equations $(20,21)$ with $\operatorname{det} Q \neq 0$. However, the equations (19) or (22) can be satisfied only if

$$
k_{1}=K_{1}, k_{2}=K_{2} \exp \left(2 \theta_{1}\right),
$$

or

$$
k_{1}=K_{1} \exp \left(\theta_{1}\right), k_{2}=K_{2} \exp \left(\theta_{1}\right),
$$

where $K_{1}, K_{2}$ are arbitrary nonzero constants.

For the case (30), i.e. for

$$
L(g)=\left(\begin{array}{cc}
K_{1} & 0 \\
0 & K_{2} e^{2 \theta_{1}}
\end{array}\right)
$$

we get

$$
P=\left(\begin{array}{cc}
1+\epsilon q_{1} & 0 \\
c_{1}\left(1+\epsilon q_{1}\right) / q_{1} & p_{2}
\end{array}\right), Q=\left(\begin{array}{cc}
q_{1} & 0 \\
c_{1} & \epsilon p_{2}
\end{array}\right) .
$$

where $\epsilon= \pm 1, c_{1}, q_{1}, p_{2}$ are arbitrary constants $q_{1} \neq 0, p_{2} \neq 0$. The equations of motion of this model read

$$
\begin{gathered}
\partial_{\nu} J^{1 \nu}=0 \\
\partial_{\nu} J^{2 \nu}+J_{\nu}^{1} J^{2 \nu}=0
\end{gathered}
$$

that actually represent the free model

$$
\begin{aligned}
& \partial_{\nu} \partial^{\nu} \theta_{1}=0 \\
& \partial_{\nu} \partial^{\nu} \theta_{2}=0
\end{aligned}
$$

For the case (31) i.e. for

$$
L(g)=\left(\begin{array}{cc}
K_{1} e^{\theta_{1}} & 0 \\
0 & K_{2} e^{\theta_{1}}
\end{array}\right)
$$

the Lax pair is given by

$$
P=\left(\begin{array}{cc}
\frac{1}{2} & 0 \\
\frac{q_{2}}{\kappa} & c_{1} \kappa
\end{array}\right), Q=\left(\begin{array}{cc}
0 & \frac{\kappa}{2} \\
c_{1} & q_{2}
\end{array}\right)
$$


where $\kappa^{2}=-K_{2} / K_{1} \neq 0$ and $c_{1}, q_{2}$ are constants, $c_{1} \neq 0$. The equations of motion of this nontrivial Af(1) model with the nonconstant metrics are

$$
\begin{gathered}
\partial_{\nu} J^{1 \nu}+\frac{1}{2}\left(J_{\nu}^{1} J^{1 \nu}-\kappa^{2} J_{\nu}^{2} J^{2 \nu}\right)=0 \\
\partial_{\nu} J^{2 \nu}=0
\end{gathered}
$$

or

$$
\begin{gathered}
\partial_{\nu} \partial^{\nu} \theta_{1}+\frac{1}{2}\left(\partial_{\nu} \theta_{1}\right)\left(\partial^{\nu} \theta_{1}\right)-\frac{1}{2} \kappa^{2} e^{-2 \theta_{1}}\left(\partial_{\nu} \theta_{2}\right)\left(\partial^{\nu} \theta_{2}\right)=0 \\
\partial_{\nu} \partial^{\nu} \theta_{2}-\left(\partial_{\nu} \theta_{1}\right)\left(\partial^{\nu} \theta_{2}\right)=0
\end{gathered}
$$

Inserting (39) into (18) we get a linear combination of the equations (40), (41) containing the parameter $q_{2}$ and we find that without loss of generality we can set $q_{2}=0$. The Lax operators for the system (40), (41) then read

$$
\begin{aligned}
& L_{0}=\partial_{0}+\left(\begin{array}{cc}
\frac{1}{2}\left(J_{0}^{1}+\kappa J_{1}^{2}\right) & \lambda\left(J_{1}^{1}+\kappa J_{0}^{2}\right) \\
0 & 0
\end{array}\right) \\
& L_{1}=\partial_{1}+\left(\begin{array}{cc}
\frac{1}{2}\left(J_{1}^{1}+\kappa J_{0}^{2}\right) & \lambda\left(J_{0}^{1}+\kappa J_{1}^{2}\right) \\
0 & 0
\end{array}\right)
\end{aligned}
$$

where $\lambda=c_{1}$ is free (spectral) parameter.

\section{Painlevé analysis and particular so- lutions}

To check the integrability of the model (42), (43) we can apply the usual Painlevé test 10], [11] i.e. to check the existence of the generic solution in the form of series with apropriate number of undetermined coefficients.

To be able to perform the test we must first convert the equations (42), (43) to the polynomial form. It can be done by the substitution $Z=\exp \left(\theta_{1} / 2\right), W=\kappa \theta_{2} / 2$ that transform the equations to the form

$$
\begin{aligned}
& Z^{3} \partial_{\nu} \partial^{\nu} Z-\left(\partial_{\nu} W\right)\left(\partial^{\nu} W\right)=0 \\
& Z \partial_{\nu} \partial^{\nu} W-2\left(\partial_{\nu} Z\right)\left(\partial^{\nu} W\right)=0
\end{aligned}
$$


The singular point analysis is trivial as all possible leading powers for the above system are nonnegative. The analysis around $Z=0$ can be done by setting $Z=1 / Y$ and consequent singular point analysis of the system

$$
\begin{gathered}
Y \partial_{\nu} \partial^{\nu} Y-2\left(\partial_{\nu} Y\right)\left(\partial^{\nu} Y\right)+Y^{6}\left(\partial_{\nu} W\right)\left(\partial^{\nu} W\right)=0 \\
Y \partial_{\nu} \partial^{\nu} W+2\left(\partial_{\nu} Y\right)\left(\partial^{\nu} W\right)=0
\end{gathered}
$$

The singular solutions of this system have leading powers of $Y$ and $W(-1,3)$ and $(-1,0)$. The resonances are $r=-3,-1,0,0$ and $r=$ $-1,0,0,3$ respectively and compatibility conditions are satisfied. (In the former case they are trivial and in the latter case we have used a Mathematica code for the check.)

The polynomial form (46), (47) of the field equations is also convenient for obtaining particular solutions of the model. Indeed, choosing $Z\left(x_{0}, x_{1}\right)=z(y), W\left(x_{0}, x_{1}\right)=w(y)$ where $y=f\left(x_{0}, x_{1}\right)$ and $f$ solving the wave equation $\partial_{\nu} \partial^{\nu} f=0$ we obtain from (46), (47) a system of ODEs for $z, w$ that is rather easy to solve. We get

$$
\begin{gathered}
z(y)=A e^{B y}+C e^{-B y} \\
w(y)=D \pm \frac{1}{2}\left(A^{2} e^{2 B y}+4 A B C y-C^{2} e^{-2 B y}\right)
\end{gathered}
$$

or

$$
z(y)=y, w(y)=C
$$

where $A, B, C, D$ are arbitrary real constants.

\section{Conclusion}

We have classified the generalized principal $A f(1)$ models that posses the Lax pair of the form (18). We have found that no such model with constant metric $L_{a b}$ in the action (5) exists and that there are just two models with the nonconstant diagonal metric. One of them is the twocomponent free model and the other one is given by the equations of motion (42), (43). A particular solution of the latter model was obtained and the Painlevé analysis confirms its integrability. 


\section{References}

[1] V.E. Zakharov and A.V. Mikhailov. Relativistically invariant two-dimensional models in field theory integrable by the inverse problem technique. J.Exp.Theor.Phys., 74(6):1953-1973, 1978. In Russian.

[2] K. Pohlmayer. Integrable hamiltonian systems and interaction through quadratic constraints. Comm. Math. Phys., 46:207-221, 1976.

[3] F. Lund. Example of a relativistic, completely integrable, hamiltonian systems. Phys.Rev.Lett., 38:1175-1178, 1977.

[4] I.V. Cherednik. Relativistically invariant quasiclassical limits of integrable two-dimensional quantum models. Theor.Math.Phys., 47(2):225-229, 1981. In Russian.

[5] N. Sochen. Integrable generalized principal chiral models. Phys.Lett. B, 391:374-380, 1997.

[6] D. Maison. Are the stationary, axially symmetric Einstein equations completely integrable? Phys. Rev. Lett, 41(8):521-522, 1978.

[7] M. Gürses and A. Karasu. Integrable sigma models. Int.J.Mod.Phys. A, 6(3):1487-500, 1991.

[8] L. Hlavatý. Towards the Lax formulation of SU(2) principal models with nonconstant metric. e-print solv-int/9908008.

[9] I.V. Cherednik. On the integrability of the equation for the twodimensional chiral asymmetric $\mathrm{O}(3)$ field and its quantum analog. Jadernaja fizika, 33(1):278-282, 1981. In Russian.

[10] M.J. Ablowitz, A. Ramani, and H. Segur. A connection between nonlinear evolution equations and ordinary differential equations of P-type. J.Math.Phys., 21:715, 1980.

[11] J. Weiss, M. Tabor, and G. Carnevale. The Painlevé property for partial differential equations. J.Math.Phys., 24:522, 1983. 